\title{
Acute kidney injury in critically ill children: incidence and risk factors for mortality
}

\author{
Sandra Mariel Martin, M.D., ${ }^{a}$ Alejandro Balestracci, M.D., ${ }^{a}$ Valeria Aprea, M.D., \\ Cecilia Bolasell, M.D., Raquel Wainsztein, M.D., ${ }^{a}$ Gustavo Debaisi, M.D., ${ }^{b}$ and \\ Gerardo Rosón, M.D. ${ }^{b}$
}

a. Nephrology Unit b. Intensive Care Unit. Hospital General de Niños "Pedro de Elizalde," Buenos Aires. Argentina

\section{E-mail Address:}

Sandra Mariel Martin: smmartin@intramed.net

Conflict of interest: None.

Received: 7-11-2012 Accepted: 27-2-2013

\begin{abstract}
Introduction. Acute kidney injury is a common complication associated with an increase in mortality in children who require intensive care. The objective of this study was to determine the incidence of acute kidney injury and identify risk factors for mortality in critically ill patients hospitalized in our facility.

Patients and Methods. This was a prospective and observational study conducted at the Intensive Care Unit (ICU) of Hospital Pedro de Elizalde between 2005 and 2009. All patients with acute kidney injury were included, and those with chronic renal failure, prerenal acute kidney injury, hepatorenal syndrome, newborn infants, and postoperative cardiovascular surgery patients were excluded.

The sample was divided into survivors and deceased patients so as to identify risk factors for mortality using univariate and multivariate analyses, taking their clinical characteristics as predictive variable, and death at the ICU as the outcome variable.

Results. Out of 1496 patients, 66 developed acute kidney injury (4.4\%). The cause was secondary in $72.8 \%$ of cases, and due to primary kidney disease in $27.2 \%$ of cases. Mortality rate was $44 \%$ ( 29 patients). The univariate analysis showed that the presence of anuria $(\mathrm{p}=0.0003$; OR: 7.01; 95\% CI: 2.3-21.35) and the need of dialysis ( $p=0.0009$; OR: 6.35 ; 95\% CI: 2.03-9.88) were significantly higher in deceased patients. The multiple regression analysis identified that the need of dialysis ( $\mathrm{p}=0.0002$; OR: $5.94 ; 95 \%$ CI: 1.85-19.04) was an independent risk factor for mortality.

Conclusions. The incidence of acute kidney injury in critically ill children was $4.4 \%$, and the need of dialysis was an independent predictor of mortality.

Key words: acute kidney injury, intensive care, incidence, mortality.
\end{abstract}

http:/ /dx.doi.org/10.5546/aap.2013.412

\section{INTRODUCTION}

Critically ill children have a higher probability of developing acute kidney injury. ${ }^{1}$

Approximately $5-12 \%$ of children hospitalized in an intensive care unit (ICU) have different degrees of acute kidney injury. ${ }^{1,2}$

In spite of the advances made in the knowledge of this condition's pathophysiology and the use of modern techniques to substitute kidney function, acute kidney injury continues extending length of stay, elevates costs of care and, above all, is associated with increased morbidity and mortality in these patients. ${ }^{13,4}$

This situation has promoted the conduction of different studies, especially in developed countries, with the purpose of identifying risk factors for mortality in critically ill children with acute kidney injury..$^{4-8}$

Initially, the lack of a consensus definition of acute kidney injury resulted in a wide variation in the incidence and mortality observed in the reported studies, thus making it hard to make comparable conclusions between the different sites.,4-8

In 2004, the Acute Dialysis Quality Initiative (ADQI) proposed to replace the term "failure" by "injury" so that physicians could focus on the early detection of kidney injury and thus prevent failure, the end stage of injury.

As a result, a classification was agreed upon for adult patients with acute kidney injury under the RIFLE criteria (risk, injury, failure, loss, end stage kidney disease), taking into account two parameters to define acute kidney injury: glomerular filtration rate and urine output. ${ }^{11}$

These criteria were subsequently validated and adapted for pediatric patients. ${ }^{12,13}$

Given that the causes of acute kidney injury vary from one country to the other ${ }^{2,4}$ and that, as far as we know, there are no local data available, we conducted this prospective and 
observational study with the following objectives: 1) to determine the incidence of acute kidney injury in children admitted to our hospital's ICU and 2) to identify, in an exploratory manner, risk factors for mortality in critically ill children with acute kidney injury.

\section{PATIENTS AND METHODS}

This prospective and observational study included all children aged from 1 month to 18 years old with acute kidney injury during their hospitalization in the ICU of Hospital General de Niños "Pedro de Elizalde" between October 2005 and September 2009.

The following patients were excluded: 1 ) newborn infants and postoperative cardiovascular surgery patients because they are more susceptible to developing acute kidney injury; ${ }^{4,14,15}$ 2) children with chronic renal failure, defined as a glomerular filtration rate lower than normal for their age, as per Schwartz formula, for more than three months; ${ }^{16} 3$ ) children with prerenal acute kidney injury, defined as a glomerular filtration rate lower than normal for their age and urinary density 1025 in the first-void urine, with a diuretic response to fluid or inotropic agent infusion for improving cardiac output within 4 hours of admission to the ICU;6,17 and 4) children with hepatorenal syndrome, defined as a glomerular filtration rate lower than normal for their age in patients with advanced liver failure, and no parenchymal renal disease, hypovolemia, shock, infection or nephrotoxicity, with no improvement following volume repletion. ${ }^{18}$

Death in the ICU was the outcome variable. Predictive variables were: age, sex, weight, height, minimum glomerular filtration rate, systemic infection, multiple organ failure, presence and length of anuria, need of dialysis, complications of dialysis, malnutrition, mechanical ventilation (MV) requirement, inotrope requirement, and length of stay at the ICU. All patients were managed by the same medical team (intensivists and nephrologists) and following the same therapeutic regimen.

\section{Definitions}

Acute kidney injury: presence of an estimated glomerular filtration rate $<50 \%$ for age, independently from diuresis, as per the pediatric RIFLE criteria. ${ }^{12,19}$ The creatinine level was measured using the Jaffe method and the glomerular filtration rate was determined using the Schwartz formula (height in $\mathrm{cm} \times \mathrm{K} /$ creatinine $[\mathrm{mg} / \mathrm{dL}]$, where $\mathrm{K}=0.45$ in infants aged 0-12 months old; 0.55 in female children and adolescents, 0.55 in male children aged 1-13 years old, and 0.70 in male adolescents aged 1318 years old)..$^{20}$

Systemic infection: documented bacteremia, known source of infection, or at least two of the following findings: idiopathic hyperventilation or hypotension, fever higher than $38^{\circ} \mathrm{C}$, or leukocytosis over $15000 / \mathrm{mm} .{ }^{3,6}$

Multiple organ failure: failure of three or more organs, excluding the kidney. ${ }^{6}$

Anuria: no urine output for a period of 24 hours. ${ }^{6}$

Malnutrition: patients with a Z-weight score under 2 standard deviations (SD). ${ }^{21}$

Indication for dialysis: severe metabolic acidosis or hyperkalemia refractory to medical treatment, volume overload and anuria for more than 24 hours.

Selection of dialysis modality: in infants and toddlers, the selected method was intermittent peritoneal dialysis, unless they had required an extensive abdominal surgery in the previous 48 hours or were hemodynamically unstable. Hemodialysis was used in children with a weight of more than $30 \mathrm{~kg}$ and hemodynamically stable, and continuous veno-venous hemodiafiltration was used in hemodynamically unstable children, not with standing their weight and age.

Complications of dialysis: internal environment disorders and infectious complications (peritonitis or vascular line-associated infections) were included.

Other complications, such as clotting of the dialysis device, filter rupture, or peritoneal catheter dysfunction were not included because, in general, they are not life-threatening.

Average length of stay in the ICU: period starting with admission to the ICU and ending on the day of death or discharge to a lower level of care within the hospital.

The study was approved by the Teaching and Research Committee and the Ethics Committee of Hospital General de Niños "Pedro de Elizalde", and all children were included in the study once their parents or adult caregiver had signed the informed consent form.

\section{Statistical Analysis}

The sample size was calculated taking into account that approximately 300 patients are discharged every year from the hospital's ICU and that the reported prevalence of acute kidney 
injury ranges from $5 \%$ to $12 \% .6,13$ Therefore, it was estimated that around 1500 patients would be included throughout the study duration (5 years) in order to identify at least 67 children with acute kidney injury with a $90 \%$ confidence level. This sample would allow to identify the risk factors strongly associated with mortality (odds ration $>6$ ).

For analysis purposes, the sample was divided into two groups: survivors and deceased patients. Continuous outcome measures did not have a normal distribution, so they were expressed as median (range), while categorical outcome measures were reported as absolute number of cases or percentage. The univariate comparison between groups for risk factors for mortality was performed using the Wilcoxon test for continuous outcome measures, and the $\chi^{2}$ test or Fisher's test for categorical outcome measures, as applicable. Variables with a value of $p<0.2$ in the univariate analysis were included in a multivariate logistic regression model in order to identify independent risk factors for mortality in critically ill children with acute kidney injury. Risk factors for mortality were quantified using odds ratio (OR) and their corresponding 95\% confidence intervals (CI), and a value of $\mathrm{p}<0.05$ was considered significant.

Data were analyzed using the Statistix 7 software (IBM version; Analytical Software, Tallahasee, FL).

\section{RESULTS}

Throughout the study period, 1496 patients were hospitalized in the hospital's ICU; out of them, 66 developed acute kidney injury, accounting for an incidence of $4.4 \%$. This group included 37 male children and 29 female children, with a median age of 2.4 years old (0.08-17.7).

Etiology was classified as renal (when the primary cause was the kidney) or extrarenal (when the kidney was secondarily affected by an extrarenal cause): 18 patients had a renal etiology and 48 , extrarenal.

The most common pathology in the first group was hemolytic uremic syndrome, while in the second group, sepsis was more frequent (Table 1).

Mortality in the total studied population was $11.8 \%$ (177 patients), whereas mortality in children with acute kidney injury was $44 \%$ (29 patients). In this group, the immediate causes of death were multiple organ failure (16 patients), septic shock (10 patients), and respiratory distress (3 patients).

At the same time, only $9(24.3 \%)$ of the surviving patients with acute kidney injury had recovered a normal renal function (glomerular filtration rate $>90 \mathrm{~mL} / \mathrm{min} / 1.73 \mathrm{~m}^{2}$ ) at the moment of discharge from the ICU.

In relation to the dialysis modality, out of the 22 patients requiring renal function replacement, 12 were treated with acute peritoneal dialysis, 3 with hemodialysis, and 4 with continuous hemodiafiltration. In three cases, more than one modality was used: two cases were first treated with hemodiafiltration and then with hemodialysis, and the third case was treated with peritoneal dialysis in addition to the other two modalities.

In order to establish the risk factors for mortality, the sample was divided into two groups: survivors $(n=37)$ and deceased patients $(n=29)$. In the comparison between both groups, the presence of anuria ( $p=0.0003$; OR: 7.01; 95\% CI: 2.3-21.35) and the need of dialysis $(\mathrm{p}=0.0009$; OR: 6.35; 95\% CI: 2.03-9.88) were

TABLE 1. Etiology and clinical course in 66 critically ill children with acute kidney injury

\begin{tabular}{lccc}
\hline Etiology & Survivors $(\mathbf{n}=\mathbf{3 7})$ & Deceased patients $(\mathbf{n = 2 9 )}$ & Total $(\mathbf{n}=\mathbf{6 6})$ \\
\hline Sepsis & 18 & 18 & 36 \\
Systemic lupus erythematosus & 3 & 3 & 6 \\
Cancer & 0 & 8 & 8 \\
Typical hemolytic uremic syndrome & 10 & 0 & 10 \\
Atypical hemolytic uremic syndrome & 2 & 0 & 2 \\
Porphyria & 1 & 0 & 1 \\
Metabolic disorder & 1 & 0 & 1 \\
Nephrotic syndrome & 1 & 0 & 1 \\
Hemophagocytic syndrome & 1 & 0 & 1 \\
\hline
\end{tabular}


significantly higher in the deceased patient group. On the contrary, there were no differences in terms of age, sex, weight, height, minimum glomerular filtration rate, systemic infection, multiple organ failure, length of anuria, length of dialysis, complications of dialysis, presence of malnutrition, MV requirement, need of inotropes, and length of stay in the ICU (Table 2).

Then, variables with a value of $p<0.2$ in the univariate analysis were incorporated into a logistic regression model, excluding the variable "presence of anuria" because it was directly related (phi coefficient of 0.91 ) to the need of dialysis.

Therefore, the multivariate analysis included the following variables: age, weight, height, and need of dialysis. As a result, only the need of dialysis continued to appear as an independent risk factor for mortality in critically ill children with acute kidney injury ( $\mathrm{p}=0.0002$; OR: $5.94 ; 95 \%$ CI: 1.85-19.04) (Table 3).

\section{DISCUSSION}

The incidence of acute kidney injury in the critically ill patients of our hospital during the study period was $4.4 \%$. In other studies, the incidence varied between $2 \%$ and $15 \%$, which reflects the differences in the characteristics of the patients assisted at the different sites..$^{2,6,8,13}$ In our study, the incidence was lower than in other studies probably because newborn infants and postoperative cardiovascular surgery patients, who have a higher risk of acute kidney injury, were excluded. ${ }^{4,14}$

The etiology was a primary kidney pathology in $27.2 \%$ of the cases, while it was caused by extrarenal conditions in $72.8 \%$, and this is consistent with the findings of other studies. $2,6,22$ As described by other authors, sepsis was the most common etiology of acute kidney injury. ${ }^{6,22}$ Besides, it is worth mentioning that in our series there was a high number of patients diagnosed with diarrhea-associated hemolytic

TABLE 2. Risk factors for mortality in 66 critically ill children with acute kidney injury. Univariate analysis

\begin{tabular}{lccc}
\hline Outcome measure & Live patients $(\mathbf{n}=37)$ & Deceased patients $(\mathbf{n}=\mathbf{2 9})$ & $\mathbf{p}$ \\
\hline Sex $(\mathrm{M} / \mathrm{F})$ & $19 / 18$ & $18 / 11$ & 0.38 \\
Age (years) & $1.75(0.08-17.25)$ & $4.36(0.33-17.75)$ & 0.07 \\
Weight $(\mathrm{kg})$ & $10.800(3-66)$ & $15(4-62)$ & 0.14 \\
Height $(\mathrm{cm})$ & $82(53-165)$ & $95(55-168)$ & 0.12 \\
Infection & 35 & 28 & 0.7 \\
Multiple organ failure & 24 & 16 & 0.42 \\
Mechanical ventilation & 26 & 19 & 0.68 \\
Inotropes & 22 & 19 & 0.61 \\
Malnutrition & 6 & 8 & 0.26 \\
Minimum glomerular filtration rate $\left(\mathrm{mL} / \mathrm{min} / 1.73 \mathrm{~m}^{2}\right)$ & $30(8-49)$ & $27(4-50)$ & 0.28 \\
Anuria & 7 & 18 & 0.0003 \\
Length of anuria & $3(1-11)$ & $4(1-37)$ & 0.66 \\
Need of dialysis & 6 & 16 & 0.0009 \\
Length of dialysis & $4.5(2-9)$ & $4(1-37)$ & 0.65 \\
Complications of dialysis & 7 & 4 & 0.74 \\
Length of stay in ICU (days) & $7(2-70)$ & $7(2-50)$ & 0.31 \\
\hline
\end{tabular}

Data are expressed as median (range) or number of cases, as applicable.

TABLE 3. Risk factors for mortality in 66 critically ill children with acute kidney injury. Multivariate analysis

\begin{tabular}{lccc}
\hline Outcome measure & $\mathbf{p}$ & Odds ratio & 95\% confidence interval \\
\hline Need of dialysis & 0.002 & 5.94 & $(1.85-19.04)$ \\
Age (years) & 0.99 & 1 & $(0.75-1.34)$ \\
Weight $(\mathrm{kg})$ & 0.25 & 0.95 & $(0.87-1.04)$ \\
Height $(\mathrm{cm})$ & 0.34 & 1.03 & $(0.97-1.08)$ \\
\hline
\end{tabular}


uremic syndrome, an expected finding due to the elevated incidence of this condition in Argentina. ${ }^{23}$

Mortality rate in critically ill children with acute kidney injury varies greatly between studies, from $25 \%$ to $80 \%$; in our study, it was $44 \% .{ }^{2,4,6-8,22,24}$ In general, mortality is primarily determined by the underlying disease and the associated hemodynamic instability. Therefore, when the kidneys are affected by sepsis or multiple organ failure, the prognosis turns grim; however, when the injury is caused by a primary renal pathology with no systemic involvement, the prognosis is more favorable. ${ }^{6,8}$ In agreement with this, all the deceased patients in our study had acute kidney injury secondary to an extrarenal pathology.

Given the known high mortality rate of critically ill children with acute kidney injury, it is pertinent to identify the predictive factors of a poor prognosis to detect those who should receive preventive and specific measures before acute kidney injury develops. These measures include maintaining an adequate renal perfusion pressure, avoiding nephrotoxic drugs, attacking infections, and ensuring that the patient has an adequate renal perfusion pressure, oxygen supply and nutrition. ${ }^{14,19,25}$ In our patients, based on the univariate analysis, the presence of anuria and dialysis requirement were significantly associated with mortality, a finding also observed by other authors. ${ }^{5,6}$

Bresolin, et al. reported that the risk of mortality increased 1.9 times when anuria developed, and 3.76 times when dialysis was needed; ${ }^{6}$ in our study, such risk went up 7 and 6.35 times, respectively. Both variables, which are directly related, are suggestive of advanced renal impairment and usually reflect the severity of the underlying disease, ${ }^{6}$ although mild presentations of acute kidney injury could also have an impact on the mortality and morbidity of the patients. ${ }^{24}$

The multivariate analysis showed that the need of dialysis was the only independent risk factor for mortality. Fifty five percent of dialysis patients died, a finding similar to that of other series. ${ }^{2,8}$ It has been proposed that starting dialysis early is associated with an improved prognosis because it avoids the complications of acute kidney injury, ${ }^{26}$ especially hypervolemia, identified as an independent risk factor for mortality in children. ${ }^{27,28}$ In support of this observation, Plötz, et al. reported that a rapid initiation of dialysis, within 24 hours of oliguria or anuria onset, has been associated with a higher survival in sepsis patients with acute kidney injury. ${ }^{29}$

Although recognizing the need of dialysis as a predictor of mortality is of prognostic significance, this clinical condition implies an advanced renal impairment, when it is no longer possible to take preventive measures. In our study, the diagnosis was based on the reduction of the glomerular filtration rate, which in turn depends on the increase of plasma creatinine. ${ }^{20}$ However, the blood creatinine level is considered to be a late parameter with little sensitivity for the diagnosis of acute kidney injury because the creatinine level is influenced by body mass, sex, age, and the underlying pathology; in addition, even small increases in the creatinine level $(0.3 \mathrm{mg} / \mathrm{dL})$ reflect a severe kidney injury associated with a poor prognosis. ${ }^{1}$ At present, in order to detect acute kidney injury early, several urine and plasma biomarkers have been identified, which are more sensitive and faster than plasma creatinine, but their clinical use is still experimental. ${ }^{1,30,31}$

This study had some limitations that are worth mentioning. First of all, the underlying condition of the deceased patients was different from that of survivors, and this could have influenced their likelihood of dying; however, the number of included patients is not enough to confirm such assumption. In addition, the sample size had an optimal power to identify only the conditions strongly associated with mortality, and other diseases with a weaker association could have been overlooked; therefore, the analysis of risk factors for mortality should be regarded as exploratory and needs to be validated by other studies including more patients.

Finally, the study was conducted in a single site and this could have led to an involuntarily selection of patients with pathologies usually seen in our hospital; however, this effect could have been lessened because many of the patients admitted to the ICU are referred from other health facilities and because the study's prospective design allowed for an analysis of all hospitalized patients throughout the study.

One last consideration to be made in relation to surviving patients is that they have a high risk of long-term renal complications. ${ }^{33}$ Askenazi ${ }^{33}$ and Hui-Stickle ${ }^{2}$ reported that between $34 \%$ and $50 \%$ of children with acute kidney injury developed chronic renal failure during follow-up. Ball and $\mathrm{Kara}^{34}$ observed that $40 \%$ of children requiring 
dialysis due to acute kidney injury had kidney alterations at the time of hospital discharge. In our study, $75 \%$ of the surviving patients were discharged from the ICU with renal impairment, and such finding highlights the importance of long-term follow-up in children with acute kidney injury.

To sum up, the incidence of acute kidney injury in our critically ill patients was $4.4 \%$, with a mortality of $44 \%$. Acute kidney injury in all deceased patients had been caused by an extrarenal pathology, and the need of dialysis was the best predictor of mortality.

\section{REFERENCES}

1. Basu RK, Chawla LS, Wheeler DS, Goldstein SL. Renal angina: an emerging paradigm to identify children at risk for acute kidney injury. Pediatr Nephrol 2012;27(7):1067-78.

2. Hui-Stickle S, Brewer ED, Goldstein SL. Pediatric ARF epidemiology at a tertiary care center from 1999 to 2001. Am J Kidney Dis 2005;45(1):96-101.

3. Lee CY, Yeh HC, Lin CY. Treatment of critically ill children with kidney injury by sustained low-efficiency daily diafiltration. Pediatr Nephrol 2012;27(12):2301-9.

4. Mehta P, Sinha A, Sami A, Hari P, et al. Incidence of acutekidney injury in hospitalized children. Indian Pediatr 2012;49(7):537-42.

5. Arora P, Kher V, Rai PK, Singhal MK, et al. Prognosis of acute renal failure in children: a multivariate analysis. Pediatr Nephrol 1997;11(2):153-5.

6. Bresolin N, Silva C, Hallla A, Toporovski J, et al. Prognosis for children with acute kidney injury in the intensive care unit. Pediatr Nephrol 2009;24(3):537-44.

7. Anochie IC, Eke FU. Acute renal failure in nigerian children: Port Harcourtexperience. Pediatr Nephrol 2005;20(11):1610-4.

8. Otukesh H, Hoseini R, Hooman N, Chalian M, et al. Prognosis of acute renal failure in children. Pediatr Nephrol 2006;21(12):1873-8.

9. Bellomo R, Ronco C, Kellum J, Mehta R, et al. Acute renalfailure -definition, outcome measures, animal models, fluid therapy and information technology needs: the Second International Consensus Conference of the Acute Dialysis Quality Initiative (ADQI) Group. Crit Care 2004;8(4):R204-12.

10. Goldstein S. Pediatric acute kidney injury: it's time for real progress? Pediatr Nephrol 2006;21(7):891-5. 11. Bellomo R. Defining, quantifying and classifying acute renal failure. Crit Care Clin 2005;21(2):223-37.

12. Akcan-Arikan A, Zappitelli M, Loftis L, Washburn K, et al. Modified RIFLE criteria in critically ill children with acute kidney injury. Kidney Int 2007;71(10):1028-35.

13. Schneider J, Khemani R, Grushkin C, Bart R. Serum creatinine as stratified in the RIFLE score for acute kidney injury is associated with mortality and length of stay for children in the pediatric intensive care unit. Crit Care Med 2010;38(3):933-9.

14. AndreoliSP. Acute kidney injury in children. Pediatr Nephrol 2009;24(2):253-63.
15. Subramanian S, Agarwal R, Deorari AK, Paul VK, Bagga A. Acute renal failure in neonates. Indian J Pediatr 2008;75(4):385-91.

16. Hogg R, FurthS, LemleyK, PortmanR, etal. National kidney foundation's kidney disease outcomes quality initiative clinical practice guidelines for chronic kidney disease in children and adolescents: evaluation, classification, and stratification. Pediatrics 2003;111(6 Pt 1):1416-21.

17. Monteverde ML, Martin SM. Insuficiencia renal aguda (IRA). En: Nefrología Pediátrica. 2a ed. Buenos Aires: Sociedad Argentina de Pediatría; 2008.Págs. 574-94.

18. Arroyo V,FernándezJ, Ginès P. Pathogenesis and treatment of hepatorenal syndrome. Semin Liver Dis 2008;28(1):81-95.

19. Martin S, Delgado N. Injuria renal aguda en pacientes críticos: Definición y clasificación actual. Medicina Infantil 2008;15(1):56-61.

20. Schwartz G, Haycock G, Edelmann C Jr, Spitzer A. A simple estimate of glomerular filtration rate in children derived from body length and plasma creatinine. Pediatrics 1976;58(2):259-63.

21. Comité Nacional de Crecimiento y Desarrollo. Guías para la evaluación del crecimiento. Buenos Aires: Sociedad Argentina de Pediatría; 2001.

22. Chang JW, Tsai HL, Wang HH, Yang LY. Outcome and risk factors for mortality in children with acute renal failure. CIin NephroI 2008;70(6):485-9.

23. Rivas M, Miliwebsky E, Chinen I, Deza N, Leotta GA. Epidemiología del síndrome urémico hemolítico en Argentina. Diagnóstico del agente etiológico, reservorios y vías de transmisión. Medicina (B Aires) 2006;66(Suppl 3):27-32.

24. Ghani AA, Al Helal B, Hussain N. Acute renal failure in pediatric patients: etiology and predictors of outcome. Saudi J Kidney Dis TranspI 2009;20(1):69-76.

25. Andreoli SP. Acute renal failure. Curr Opin Pediatr 2002;14(2):183-8.

26. Fernández C, López-Herce J, Flores JC, Galaviz D, et al. Prognosis in critically ill children requiring renal replacement therapy. Pediatr Nephrol 2005;20(10):1473-7.

27. Gillespie RS, Seidel K, Symons JM. Effect of fluid overload and dose of replacement fluid on survival in hemofiltration. Pediatr Nephrol 2004;19(12):1394-9.

28. Foland JA, Fortenberry JD, Warshaw BL, Pettignano R, et al. Fluid overload before continuous hemofiltration and survival in critically ill children: a retrospective analysis. Crit Care Med 2004;32(8):1771-6.

29. Plötz FB, Hulst HE, Twisk JW, B6kenkamp A, et al. Effect of acute renal failure on outcome in children with severe septic shock. Pediatr NephroI 2005;20(8):1177-81.

30. Goldstein SL. Acutekidney injury biomarkers: renal angina and the need for a renal troponin I. BMC Med 2011;9:135.

31. Al-Ismaili Z, Palijan A, Zappitelli M. Biomarkers of acute kidney injury in children: discovery, evaluation, and clinical application. Pediatr NephroI 2011;26(1):29-40.

32. Goldstein SL, Devarajan P. Acute kidney injury in childhood: should we be worried about progression to CKD? Pediatr NephroI 2011;26(4):509-22.

33. Askenazi DJ, Feig DI, Graham NM,Hui-StickleS, Goldstein SL. 3-5 year longitudinal follow-up of pediatric patients after acute renal failure. Kidney Int 2006;69(1):184-9.

34. Ball EF, Kara T. Epidemiology and outcome of acute kidney injury in New Zealand children. J Paediatr Child Health 2008;44(1):642-6. 